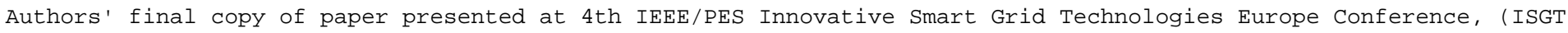

Europe), 2013, Oct 6-9, Copenhagen, Denmark.

\title{
Applying Model Predictive Control to Power System Frequency Control
}

\author{
Anne Mai Ersdal*, Inês M. Cecílio ${ }^{\dagger}$, Davide Fabozzi ${ }^{\dagger}$, Lars Imsland* and Nina F. Thornhill ${ }^{\dagger}$ \\ ${ }^{*}$ Department of Engineering Cybernetics \\ Norwegian University of Science and Technology, Trondheim, Norway \\ \{anne.mai.ersdal, lars.imsland\}eitk.ntnu.no \\ ${ }^{\dagger}$ Department of Chemical Engineering \\ Imperial College, London, United Kingdom \\ \{i.cecilio09,d.fabozzi,n.thornhill\}@imperial.ac.uk
}

\begin{abstract}
Model predictive control (MPC) is investigated as a control method which may offer advantages in frequency control of power systems than the control methods applied today, especially in presence of increased renewable energy penetration. The MPC includes constraints on both generation amount and generation rate of change, and it is tested on a one-area system. The proposed MPC is tested against a conventional proportionalintegral (PI) controller, and simulations show that the MPC improves frequency deviation and control performance.
\end{abstract}

\section{INTRODUCTION}

One important control aspect of a power system is to balance power demand and supply in a cost efficient and flexible manner. This is often referred to as frequency control (FC) or balancing control; the former term will be applied here. Today, many interconnected power systems use a hierarchical control structure of three levels to maintain this balance. These three levels, in increasing hierarchical order, are often referred to as primary, secondary and tertiary control [2]. Equivalent, less ambiguous terms have recently been proposed: frequency containment reserve (FCR), frequency restoration reserve (FRR) and replacement reserve ( $\mathrm{RR})[3]$.

FCR controllers are local, automatic controllers situated at the generating units. The FRR controller is a centralized, often automated controller that deals with determining the combination of available generating units to satisfy the power demand. This service is also referred to as automatic generation control (AGC) or load frequency control (LFC). Additionally, RR control, which is supervisory to FRR control, is mainly executed manually by the transmission system operators (TSOs).

In the Nordic network, the hydro power generators have historically been the main provider of FCR, while other generating units such as thermal and nuclear power generators as well as some controllable loads participate in RR [4]. In December 2012, the Norwegian operator Statnett became the first Nordic TSO to adopt FRR [4]: the other operators have introduced similar services in early 2013. It is likely that both hydro and thermal power generators will provide FRR.

The financial support from the Marie Curie FP7-IAPP project "Using realtime measurements for monitoring and management of power transmission dynamics for the Smart Grid - REAL-SMART" [1] Contract No: PIAPGA-2009-251304, the Portuguese Foundation for Science and Technology (FCT) under Fellowship SFRH/BD/61384/2009, and the Research Council of Norway project 207690 "Optimal Power Network Design and Operation" are gratefully acknowledged.

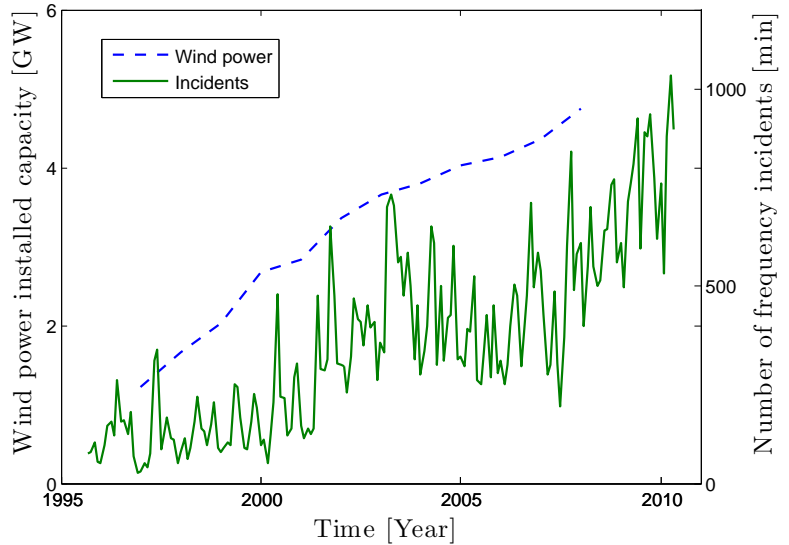

Figure 1: Wind power installed capacity [7] and number of frequency incidents per month in the Nordic system [8].

Recently, there have also been suggestions on how to include industrial loads in FRR schemes [5].

Proportional-integral (PI) controllers are the state-of-the-art choice for FC, and these are tuned based on operator practice [6]. Fig. 11 shows how the number of frequency incidents, i.e. minutes spent outside $49.9-50.1 \mathrm{~Hz}$, has developed concurrently with the wind power penetration in the Nordic network during the last decade. It is clearly increasing, and it can be assumed that today's FC methods are losing their suitability for future power systems. One of the main problems is their ability to deal with the intermittency associated with renewable energy resources, especially wind power.

Since energy cannot be stored efficiently, the power from the generating units is fed directly into the grid. The unpredictable nature of renewable resources thus results in greater fluctuations in the power supply. This introduces challenges with regards to $\mathrm{FC}$, and it is becoming increasingly difficult to guarantee a stable and reliable power supply.

Based on these challenges, there has been an increasing interest in applying MPC for FC during the last decade, some examples being [9]-[11]. The majority of work that has been published concerns multi-area systems where controlling the power flow between the areas is included in the control objective, and they exclusively apply linear MPC. In this work 
the MPC is non-linear, which means that it easily allows for extension to more complicated non-linear models, and the frequency-control hierarchy is also viewed separately with distinct governor and turbine models for each level.

\section{SySTEM MODEL}

\section{A. System Equations}

The model used in this paper is inspired by [6]. It is a onearea model where network and electromechanical dynamics are neglected. The choice of model allows for clear analysis of the effect of the MPC without the complications of voltage dynamics and inter-area oscillations. The dynamics of the entire one-area system is represented through the following differential equation [6]

$$
\frac{d \Delta f}{d t}=\frac{1}{2 H}\left(\Delta P_{m}-\Delta P_{L}\right)-\frac{D}{2 H} \Delta f
$$

where $\Delta f$ is the deviation from the nominal frequency $f_{s}$, $\Delta P_{m}$ the total change in mechanical power from primary, secondary and tertiary control combined, $\Delta P_{L}$ the electrical power imbalance, $H$ the inertia of the rotating masses, and $D$ the load damping coefficient of the network.

In addition there are equations representing the governors and turbines. The turbines participating in FCR are modeled as simplified hydro turbines [2]

$$
\begin{aligned}
& \text { Governor: } \Delta P_{g}(s)=\frac{1+T_{g 2} s}{\left(1+T_{g 1} s\right)\left(1+T_{g 3} s\right)} \cdot \Delta P_{c}(s) \\
& \text { Turbine: } \Delta P_{m}(s)=\frac{1-T_{t} s}{1+0.5 T_{t} s} \cdot \Delta P_{g}(s)
\end{aligned}
$$

and the FRR and RR-turbines as generic thermal units [6]

$$
\begin{aligned}
& \text { Governor: } \Delta P_{g}(s)=\frac{1}{1+T_{g} s} \cdot \Delta P_{c}(s) \\
& \text { Turbine: } \Delta P_{m}(s)=\frac{1}{1+T_{t} s} \cdot \Delta P_{g}(s)
\end{aligned}
$$

where $\Delta P_{c}$ is the control signal to the governor, $\Delta P_{g}$ the valve position from the governor to the turbine, $\Delta P_{m}$ the mechanical power output from the turbine, and $T_{g}$ and $T_{t}$ the time constants of the governor and turbine, respectively.

The local FCR controllers are implemented as proportional controllers

$$
\Delta P_{c, F C R}=-\frac{1}{R} \operatorname{sat}(\Delta f)
$$

where $R$ is the droop given by the TSO, and sat $(\Delta f)$ is $\Delta f$ saturated at \pm 0.1 . This is done in order to model full FCR activation at frequency deviations outside $\pm 0.1 \mathrm{~Hz}$.

The total system can therefore be represented by a nonlinear dynamic equation

$$
\dot{x}=f(x, u, w)
$$

Depending on the number of governors/turbines $m$ used to represent each control level, the system consists of $7 m+1$ state variables $x=\left[\begin{array}{lllll}\Delta f & x_{g t}^{1} & x_{g t}^{2} & \cdots & x_{g t}^{m}\end{array}\right]^{T}$, where $x_{g t}^{i}=$ $\left[\begin{array}{llllll}x_{1}^{i} & x_{2}^{i} & x_{3}^{i} & \Delta P_{g, F R R}^{i} \Delta P_{m, F R R}^{i} \Delta P_{g, R R}^{i} \Delta P_{m, R R}^{i}\end{array}\right]^{T}, 2 m$ controlled inputs $u^{i}=\left[\begin{array}{ll}\Delta P_{c, F R R}^{i} & \Delta P_{c, R R}^{i}\end{array}\right]^{T}$, and one disturbance $w=\Delta P_{L}$. The state variables $x_{1}^{i}, x_{2}^{i}$ and $x_{3}^{i}$ are the state variables from the hydro turbine and governor transfer functions, of which $\Delta P_{g, F C R}$ and $\Delta P_{m, F C R}$ can be found.

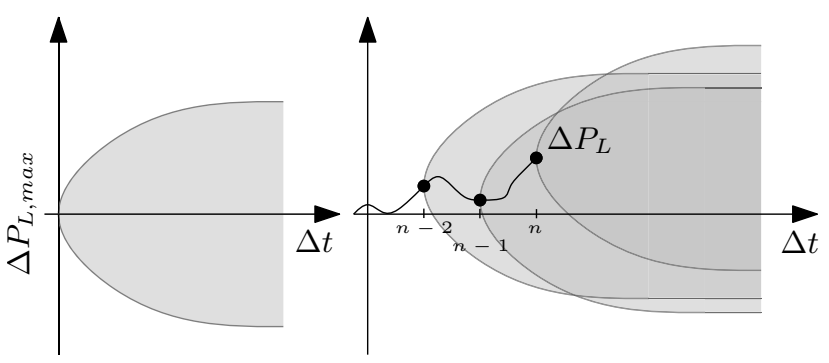

Figure 2: Area defining the possible worst-case estimated power imbalance $\Delta P_{L, \max }$ (left), and its evolution (right).

\section{B. System disturbance $\Delta P_{L}$}

Viewing only the unpredicted power imbalance as a disturbance, the main disturbance with regards to $\mathrm{FC}$ is the difference between power supply and power consumption from intermittent generators and loads, respectively. This can be summarized as one total unpredicted load-power imbalance $\Delta P_{L}$.

In power networks including a certain amount of wind power, such as the Nordic grid, this unpredicted power imbalance can for simplicity be assumed to be dominated by the fluctuations in produced wind power. For different wind farms the worst case variations from the predicted power output can be estimated, for example [12] presents worstcase variations for a specific wind farm within one second, one minute and one hour into the future $\Delta t$. Points from several wind farms may be combined and interpolated to create a worst-case estimate for the continuous-time bound of the power imbalance. Fig. 2 shows how such an estimate could look, and also displays how the worst-case variation following present time $n$ is restricted by the curve at time $n$ as well as the curves of previous time instants $n-1, n-2$ and so on. In Fig. 2 this corresponds to the most shaded intersection area between the three curves.

\section{System Delays and Control Signal Dispatching}

There are several delays in a power system, and the ones included in this model are presented in the following. First is the delay of the signal into the FRR and RR controllers. This is due to delays associated with performing measurements and state estimation, and it is set to $40 \mathrm{~s}$. Then there are the delays which represent the time it will take for the different turbines to deliver what they are asked. In this work, such delays are included in the FRR and RR, and they are set to $20 \mathrm{~s}$ and 120 $\mathrm{s}$, respectively. All of these delays are known to the MPC. There are no delays associated with the FCR, as its control is based on local frequency measurements and it is required to act instantaneously.

The FCR control signal is continuous, but the FRR and $\mathrm{RR}$ control signals are dispatched at certain intervals and kept constant between the dispatching times. The FRR and RR control signals are dispatched every 10 and 60 s, respectively.

\section{CONTROller}

\section{A. Control Problem}

The main purpose of the three controllers is to keep the frequency at a level where the system operation is safe and 


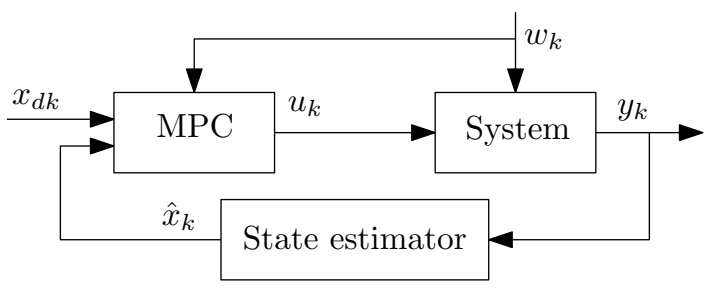

Figure 3: Overview of MPC-loop.

stable. In the Nordic network this is considered to be between 49.9 and $50.1 \mathrm{~Hz}$. However, the costs of using the reserves are not equal in the three cases. The FCR is the most expensive and the RR the least expensive, in accordance to how fast they are required to activate. So another incentive for the controller is to keep the total cost associated with FC at a minimum.

\section{B. $M P C$}

An MPC will be applied for FRR and RR control of this system. MPC is a framework for advanced control that has seen widespread use in other industries [13], [14], and it is believed that its optimizing and constraint-handling properties make it promising for FC.

One of the strengths of an MPC is that it can exploit the knowledge one might have about the disturbances affecting the system. This is something not usually done with the PI control methods presently being used.

MPC uses a model of the system to predict how it will behave in the future, and then optimizes the controlled input with regards to an objective function. Mathematically, it can be formulated as a continuous time optimal control problem on the form (6a) subjected to 6b - 6d [15]

$$
\begin{aligned}
\min _{x(\cdot), u(\cdot)} J(x(t), u(t)) & \text { Fixed initial state } \\
x(0)-x_{0} & =0 \text { System model } \\
\dot{x}(t)-f(x(t), u(t)) & =0 \text { S } \\
g(x(t), u(t)) & \leq 0 \text { Path constraints }
\end{aligned}
$$

where $x(t)$ are the system states, $u(t)$ the system controlled inputs, $w(t)$ the system disturbances, and $J(x(t), u(t))$ the control objective function.

Fig. 3 shows the basics of how an MPC works. The idea is to solve an optimization problem at each time step to find the optimal system input over a fixed time horizon $u(t)$ with respect to the objective function $J(\cdot)$, and then apply the first element of $u(t)$ as input to the system. The loop is closed by the measurements $y(t)$, and a state estimator is also included as the MPC needs knowledge of the entire state vector $x(t)$. Here it will be assumed that the entire state vector is known. In the case where one wants the state to follow a (possibly time varying) reference, the desired state reference is given as $x_{d}(t)$. In this case we want $\Delta f$ to go to zero, and $x_{d}(t)$ hence consists of zeros.

The MPC will be based on the model given by (5). The saturation of (4) is nonlinear, implying that nonlinear MPC (NMPC) must be used. The path constraints includes limita-

\begin{tabular}{|c|c|c|c|}
\hline $\mathbf{H}$ & D & $\mathbf{R}$ & $f_{s}$ \\
\hline $0.0835 \mathrm{pu} \cdot \mathrm{s}$ & $0.045 \mathrm{pu} / \mathrm{Hz}$ & $3 \mathrm{~Hz} / \mathrm{pu}$ & $50 \mathrm{~Hz}$ \\
\hline $\mathbf{T}_{\mathrm{g} 1, \mathrm{FCR}}$ & $\mathbf{T}_{\mathrm{g} 2, \mathrm{FCR}}$ & $\mathbf{T}_{\mathrm{g} 3, \mathrm{FCR}}$ & $\mathbf{T}_{\mathbf{t}, \mathbf{F C R}}$ \\
\hline $0.5 \mathrm{~s}$ & $3 \mathrm{~s}$ & $50 \mathrm{~s}$ & $0.5 \mathrm{~s}$ \\
\hline $\mathbf{T}_{\mathbf{g}, \text { FRR }}$ & $\mathbf{T}_{\mathbf{t}, \mathbf{F R R}}$ & $\mathbf{T}_{\mathrm{g}, \mathrm{RR}}$ & $\mathbf{T}_{\mathbf{t}, \mathbf{R R}}$ \\
\hline $0.08 \mathrm{~s}$ & $30 \mathrm{~s}$ & $0.08 \mathrm{~s}$ & $40 \mathrm{~s}$ \\
\hline$\left|\Delta \mathbf{P}_{\mathbf{c}, \mathbf{F R R}}\right|$ & $\left|\Delta \mathbf{P}_{\mathbf{c}, \mathbf{R R}}\right|$ & $\left|\Delta \dot{\mathbf{P}}_{c, \text { FRR }}\right|$ & $\left|\Delta \dot{\mathbf{P}}_{\mathbf{c}, \mathbf{R R}}\right|$ \\
\hline$\leq 0.05 \mathrm{pu}$ & $\leq 0.25 \mathrm{pu}$ & $\leq 0.0002 \mathrm{pu} / \mathrm{s}$ & $\leq 0.0001 \mathrm{pu} / \mathrm{s}$ \\
\hline
\end{tabular}
tions on the generation capacity and limitations on the rate of change in generation.
Table I: System parameters.

The continuous time optimization problem (6a) in the MPC is solved with direct methods, that is, it is discretized and transformed into a nonlinear program (NLP) [15]. In this work collocation has been applied for discretization, and it has been implemented using the CasADi software [16].

\section{Simulations}

\section{A. Case Study}

The frequency response of the power system implemented in CasADi was validated by using the FRECOL packag $\mathrm{q}^{2}$ One governor and turbine per control level is implemented $(m=1)$, and the system parameters can be seen in Table I The constraints on the input $\left|\Delta P_{c}\right|$ and the input rate of change $\left|\Delta \dot{P}_{c}\right|$ are also given in Table I . which refer to a per-unit base of $10 \mathrm{GW}$.

\section{B. Tuning the $M P C$}

The main tuning variables of the MPC are the prediction horizon for the optimization $T$ and the design of the objective function $J(\cdot)$. The continuous time objective function was set to

$$
J(x, u)=\int_{t=0}^{T} x^{T} Q x+u^{T} R u+\dot{u}^{T} S \dot{u} d t
$$

where $Q, R$ and $W$ are real, symmetric and positive semidefinite tuning matrices. These matrices are often chosen to be diagonal [19], and their values are chosen such that deviation from zero of certain state variables, and the magnitude and change of inputs are punished in a desirable way. Here, the elements of these tuning matrices were chosen to be $q_{11}=1$, $q_{i j}=0$ for $i, j \neq 1, r_{11}=0.01, r_{k l}=0$ for $k, l \neq 1$, $s_{11}=0.1, s_{k l}=0$ for $k, l \neq 0$, where $i, j=1, \cdots, 8$ and $k, l=1, \cdots, 2$. The optimization horizon $T$ was chosen to be 5 minutes, a decision based on a compromise between system time constants and complexity.

\section{Comparison Case, PI-controller}

The performance of the MPC is compared with the performance of a generic PI-controller [18]. There the FRRcontroller is a PI-controller

$$
\Delta P_{c, F R R}=K_{p} \Delta f+K_{i} \int \Delta f d t
$$

\footnotetext{
${ }^{2}$ Acronym for Frequency REserve Control Open-source Library [17 developed in the course of research reported in 18
} 


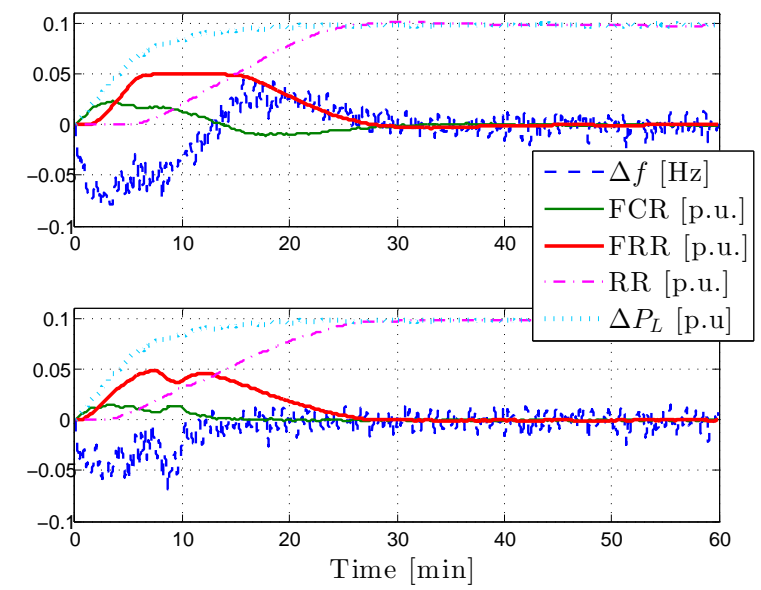

Figure 4: Case B. Above: PI-controller. Below: MPC.

and the RR-controller is an I-controller which takes in $\Delta P_{c, F R R}$ as input

$$
\Delta P_{c, R R}=K_{i i} \int \Delta P_{c, F R R} d t
$$

Both the FRR and RR control signal are limited by their respective generation capacity and limits on the generation rate of change. The control parameters of the PI-controller are $K_{p}=-0.015, K_{i}=-0.003$ and $K_{i i}=0.002$.

The two controllers are compared against each other by applying a control performance measure (CPM). In [20] two control performance criteria for FC are presented, which are more general formulations of the performance criteria used by the North American electric reliability corporation (NERC). These are used to ensure a certain quality of the FC by demanding that they stay below a certain value, and they can therefore also be used as a CPM: the lower the CPM, the better. The solution for one-area systems from [20] was used, where the average of $\Delta f$ is calculated over windows of $30 \mathrm{~s}$ in order to filter out the fast fluctuations. The CPM is then found by again taking the average of all these windows.

\section{Simulations}

1) Case A: In case A the MPC is tested with a $\Delta P_{L}$ that follows the positive worst-case scenario from Fig. 2. Fig. 4 shows the simulation results, note that it is $\Delta P_{m}$ which is plotted for FCR, FRR and RR. In this case it is clearly seen that the MPC keeps $\Delta f$ closer to zero than the PI-controller, and that the use of FCR and FRR are lowered, while the use of RR is slightly increased.

2) Case $B$ : In Case $\mathrm{B} \Delta P_{L}$ first follows the positive worst-case scenario, and then changes direction to follow the negative worst-case scenario after approximately $900 \mathrm{~s}$. The simulation results can be seen in Fig. 5. As in Case B it is clearly seen from Fig. 5 that $\Delta f$ is kept closer to zero, and that the use of all three reserves is reduced.

3) Several cases: Case A and B concerned constructed situations where the difference between the PI-controller and the MPC are clearly seen. Here, a collection of 200 simulations

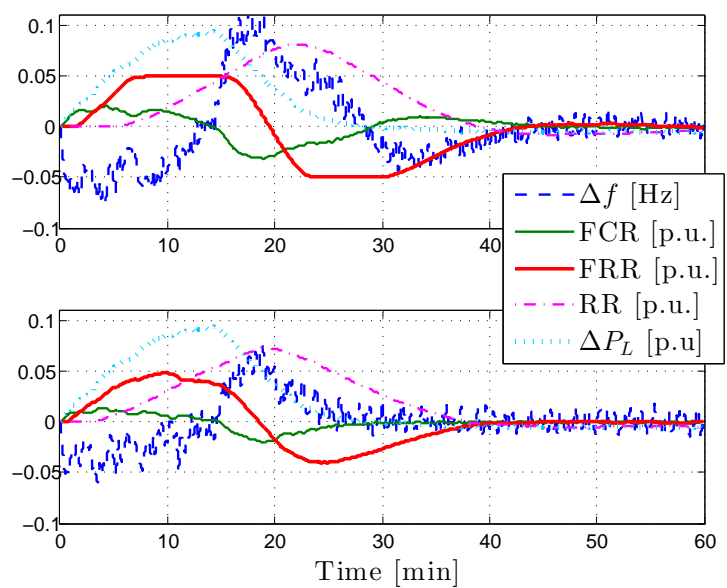

Figure 5: Case C. Above: PI-controller. Below: MPC.

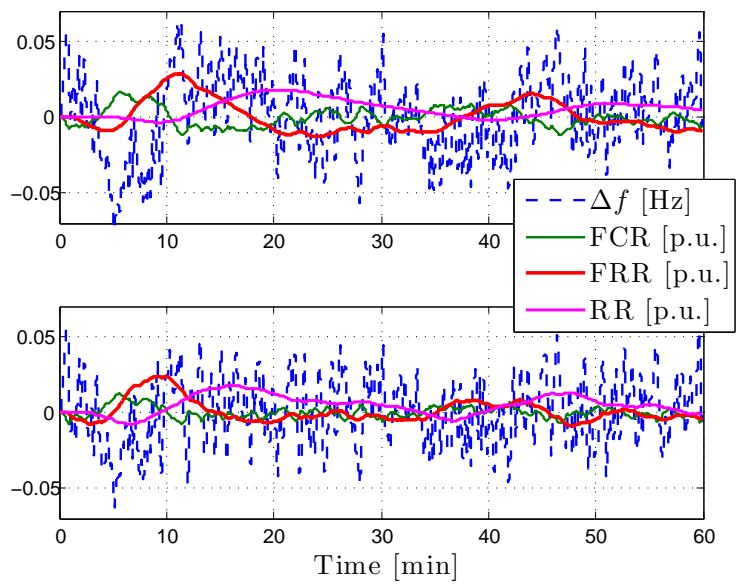

Figure 6: Case A1. Above: PI-controller. Below: MPC.

with more realistic, random $\Delta P_{L}$ restricted to the worst-case area of Fig. 2 is considered. Each simulation lasts for 60 minutes. The general trend is that the frequency response with the PI-controller and the MPC are similar, with the MPC performing somewhat better judging by the CPM. A decrease in use of FCR and FRR is also observed with the MPC, while the use of RR is increased. These trends are reflected in Fig. 6 , which displays one of the 200 simulations made. The average CPM and use of reserves can be found in Table $\amalg$

Table II: Average CPM and reserves usage (in MWh).

\begin{tabular}{ccccc}
\hline & CPM $\left(\cdot 10^{-4}\right)$ & FCR & FRR & RR \\
\hline PI & 4.8 & 22 & 43 & 39 \\
\hline MPC & 3.1 & 15 & 27 & 51 \\
\hline Difference & $-35 \%$ & $-32 \%$ & $-37 \%$ & $+31 \%$ \\
\hline
\end{tabular}




\section{Discussion}

Extensive simulation supports that the MPC does perform better than the PI (Table II). A reduction in use of the expensive FCR and FRR is also achieved, with higher use of the cheaper RR.

One important reason that the MPC is performing better is the knowledge the disturbance measurement brings to the controller. It should be emphasized that the MPC has no knowledge of how the disturbance $\Delta P_{L}$ will develop in the future. It is provided with an accurate measurement of the disturbance at the present time, and this is then assumed to be constant for the entire optimization horizon. The knowledge of the current disturbance causes the MPC to react faster, and also helps it to plan ahead.

The MPC also tries to minimize the use of FRR (through $r_{11} \cdot \Delta P_{c, F R R}^{2}$ in the objective function), which helps to keep FRR from staying saturated over longer periods. This is especially visible in Case A and B, where the FRR with PI-control stays saturated over a longer period (see Fig. 4 and 5), and it is beneficial since it releases FRR to act in the case of new incidents. The small oscillation in the FRR of Fig. 4 shows how the MPC balances the use of FRR against the size of $\Delta f$. This is a direct result of the weights put on $\Delta f$ and $\triangle P_{c, F R R}$ in the objective function.

Another reason for the improvement seen when using MPC is that the FRR becomes more versatile, and also that the control actions behind the FRR and RR are coordinated. Again this is clearly seen in Case $\mathrm{A}$ and $\mathrm{B}$, where the overshoot $\Delta f$ is experiencing with the PI-controller is removed (see Fig. 4 and 5). This is because the usage of FRR and RR are coordinated to ensure a smooth convergence of $\Delta f$ to zero, and hence avoiding using unnecessary FCR.

It should be mentioned that the way the PI-controller implements the RR is a simplification of the real world. As mentioned in Section I the RR control is executed manually by the TSO. However, in order to compare the MPC with the PI-controller, the RR controller had to be modeled, and it is the authors' opinion that the way of Section IV-C is satisfactory for the scope of this work.

The fact that the PI and the MPC have similar performance over 200 different $\Delta P_{L}$ implies that the PI-controller is well tuned. However, it might still be argued that the PI-controller could be tuned in a different way to achieve a behavior more similar to the MPC, for instance the RR could be more aggressive in Case A. On the other hand, this could lead to a controller with worse performance in a situation such as Case B. The MPC on the other hand does not need to be retuned for it to handle both situations better than the PI-controller.

In this paper, the model applied in the MPC and the one used for simulation are identical, and this is of course one reason for the success of the MPC. However the work presented here still shows how the MPC can use disturbance measurement and model information to give improved input flexibility and coordination, and it is believed that these features will be prominent also when model mismatch is included.

\section{CONCLUSION AND FURTHER WORK}

The work presented in this paper shows that MPC is promising with regards to FC. It it shown through different examples that using an MPC for FC can both improve the performance with regards to frequency deviation, but also lower the use of the more expensive FCR and FRR at the expense of higher use of the cheaper RR.

Even though some simplifications have been made (one is identity between the model applied in the MPC and the simulation model), the results presented here still shows some of the benefits an MPC provides with regards to exploiting disturbance knowledge, planning ahead, and coordinating system inputs. A natural extension to this paper is to eliminate some of these simplifications, for example adding model mismatch or including several turbines per control level. Note that the framework allows for extensions with nonlinear models.

Other plans for further work is to look at a robust MPC which takes into account three possible future developments of the disturbance, one neutral, one towards the positive worstcase and one towards the negative worst-case scenario.

\section{REFERENCES}

[1] REAL-SMART project. http://www3.imperial.ac.uk/realsmart, accessed February 2013

[2] J. Machowski, J. W. Bialek, and J. R. Bumby, Power System Dynamics. Stability and Control. Wiley, 2008.

[3] Operational Reserve Ad Hoc Team Report. v. 6, ENTSO-E, May 2012.

[4] Statnett, "Systemdrifts- og markedsutviklingsplan 2012," tech. rep., 2012.

[5] D. Fabozzi, N. F. Thornhill, and B. C. Pal, "Frequency restoration reserve control scheme with participation of industrial loads," in Proc. 2013 IEEE Grenoble PowerTech conf., pp. 1-6, June 2013.

[6] H. Bevrani, Robust Power System Frequency Control. Springer, 2009.

[7] ENTSO-E Annual Statistics. https://www.entsoe.eu/publications/formerassociations/nordel/annual-statistics/, accessed March 2013.

[8] D. Whitley and O. Gjerde, "LFC/AGC-Nordic and European perspective," in Exchange of balancing services between the Nordic and the Central European synchronous systems, pp. 1-13, 2011.

[9] A. N. Venkat, I. A. Hiskens, J. B. Rawlings, and S. J. Wright, "Distributed MPC Strategies With Application to Power System Automatic Generation Control,' IEEE Transactions on Control Systems Technology, vol. 16, pp. 1192-1206, Nov. 2008.

[10] M. Shiroei, A. M. Ranjbar, and T. Amraee, "A functional model predictive control approach for power system load frequency control considering generation rate constraint," International Transactions on Electrical Energy Systems, vol. 23, pp. 214-229, 2013.

[11] T. H. Mohamed, J. Morel, H. Bevrani, and T. Hiyama, "Model predictive based load frequency control design concerning wind turbines," International Journal of Electrical Power \& Energy Systems, vol. 43, pp. 859-867, Dec. 2012.

[12] H. Holttinen, The impact of large scale wind power production on the Nordic electricity system. $\mathrm{PhD}$ thesis, Helsinki University of Technology, 2004.

[13] L. Magni, D. M. Raimondo, and F. Allgöwer, Nonlinear Model Predictive Control. Towards New Challenging Applications. Springer, 2009.

[14] E. D. Mehleri, L. G. Papageorgiou, N. C. Markatos, and H. Sarimveis, "A model predictive control framework for residential microgrids," Computer Aided Chemical Engineering, vol. 30, pp. 327-331, 2012.

[15] L. T. Biegler, Nonlinear Programming: Concepts, Algorithms, and Applications to Chemical Processes. SIAM, 2010.

[16] J. Andersson, J. Åkesson, and M. Diehl, "CasADi - A symbolic package for automatic differentiation and optimal control," in Recent Advances in Algorithmic Differentiation (S. Forth, P. Hovland, E. Phipps, J. Utke, and A. Walther, eds.), vol. 87 of Lecture Notes in Computational Science and Engineering, pp. 297-307, Springer Berlin Heidelberg, 2012.

[17] "FRECOL project." http://www.frecol.co.uk accessed June 2013.

[18] I. M. Cecílio, A. M. Ersdal, D. Fabozzi, and N. F. Thornhill, "An opensource educational toolbox for power system frequency control tuning and optimization." Submitted to 4th IEEE PES Innovative Smart Grid Technologies (ISGT) Europe Conference, 2013.

[19] J. B. Rawlings and D. Q. Mayne, Model Predictive Control: Theory and Design. Nob Hill Publishing, 2009.

[20] G. Gross and J. W. Lee, "Analysis of load frequency control performance assessment criteria," IEEE Transactions on Power Systems, vol. 16, pp. 520-525, 2001. 